\title{
LA INTERPRETACIÓN DE SINTAGMAS PREPOSICIONALES ESCUETOS INTRODUCIDOS POR LA PREPOSICIÓN EN*
}

\author{
InÉs KugueL \\ Universidad General Sarmiento, \\ Universidad de Buenos Aires \\ ikuguel@ungs.edu.ar
}

\author{
Carolina Oggiani \\ Universidad de Buenos Aires, \\ Universidad de la República \\ oggiani.carolina@gmail.com
}

In this paper we deal with some locative expressions from Río de la Plata Spanish in which the preposition en combines with singular count nominals. We examine their semantic and syntactic behavior by applying a series of tests and, as a result, we classify them according to three semantic classes. In the first class, the bare noun has a generic reading (Los alumnos suelen estudiar en biblioteca). Regarding the second class, the bare noun refers to an individual entity (La reunión de los claustros se hace a las 17 horas en rectorado), while in the third class the bare nominal refers to a prototypical activity that takes place in the location denoted by the noun (En este momento, el doctor está en quirófano). Furthermore, we analyze some uses that show microvariation between Uruguayan and Argentinian Spanish. Finally, we claim there is a possible tendency

* La primera versión de este trabajo fue presentada en el workshop VII Romania Nova, que tuvo lugar en Buenos Aires del 26 al 28 de noviembre de 2014. Agradecemos los comentarios y sugerencias de los dictaminadores anónimos, que fueron de suma importancia para su mejora. 
in colloquial Río de la Plata Spanish towards allowing a wider distribution of bare nominals than those previously attested in the literature (Bosque 1996; Masullo 1996; Laca 1999; Espinal 2010; Espinal \& McNally 2011).

Keywords: prepositional phrase, bare nominals, dialectal microvariation, Río de la Plata Spanish, semantics

En este trabajo analizamos un grupo de construcciones locativas, propias del español rioplatense, en las que la preposición en se combina con nombres singulares contables sin determinante. Presentamos una serie de pruebas, con el fin de explorar su comportamiento semántico y sintáctico y, además, elaboramos una clasificación que agrupa estas construcciones de acuerdo con tres clases semánticas. Proponemos que en el primer grupo de locativos (Los alumnos suelen estudiar en biblioteca) el nombre escueto adquiere una lectura genérica. Por su parte, en el segundo grupo (La reunión de los claustros se hace a las 17 horas en rectorado) el escueto refiere a una entidad individual; mientras que en el tercer grupo (En este momento, el doctor está en quirófano), el escueto denota una actividad prototípica llevada a cabo en la localización designada por el nombre. Asimismo, en este trabajo mostramos algunos casos de microvariación dialectal y distinguimos usos propios del español de Uruguay, distintos a los del español de Argentina. Finalmente, proponemos algunas reflexiones respecto de una posible tendencia general del español rioplatense que permite la presencia de nombres escuetos con más valores y mayor distribución de lo que la literatura ha seńalado hasta el momento para el español general (Bosque 1996; Masullo 1996; Laca 1999; Espinal 2010; Espinal \& McNally 2011).

Palabras clave: sintagma preposicional, nombres escuetos, microvariación dialectal, español rioplatense, semántica 


\section{INTRODUCCIÓN}

En este trabajo analizamos un grupo de construcciones locativas introducidas por la preposición en, propias de la variedad del español rioplatense, ${ }^{1}$ como las que ilustramos en (1).

(1) a. Los alumnos suelen estudiar en biblioteca.

b. La reunión de los claustros se hace a las 17 horas en rectorado.

c. En este momento, el doctor está en quirófano.

El interés de estas construcciones radica en que han sido poco exploradas en español. Si bien existen numerosos antecedentes en otras lenguas, como en inglés y holandés, que se detienen en el comportamiento de los sintagmas preposicionales escuetos (Baldwin et al 2006; Carlson 2006; Stvan 2009; Le Bruyn et al 2011), en español este fenómeno ha recibido menos atención (cf. Bosque 1996; Masullo 1996; Laca 1999). La literatura se ha ocupado sobre todo de escuetos en posición de objeto que denotan propiedades, es decir, sin valor argumental (cf. Bosque 1996; Espinal 2010; Espinal \& McNally 2011). Como veremos, solo en algunos casos se han señalado usos preposicionales propios del español americano y más en particular, del español rioplatense (RAE/ASALE 2009).

${ }^{1}$ Con el término "español rioplatense" nos referimos a las propiedades en común que tienen las variedades dialectales habladas en ambas orillas del Río de la Plata -la argentina y la uruguaya. Resulta relevante aclarar que el español rioplatense incluye a las ciudades capitales de Buenos Aires y Montevideo. La variedad que se usa en ellas es la que prevalece en los medios gráficos y audiovisuales de circulación masiva en ambos países y la que se constituye en lengua estándar. 
Los casos en los que nos detendremos son aquellos en los que aparece un nombre contable singular sin determinante en un sintagma preposicional encabezado por la preposición en y que tienen valor locativo. Así, quedan excluidas de este análisis las secuencias con en u otras preposiciones que presentan valor temporal (en primavera) o modal (a mano) y las que denotan medios de locomoción (en tren), instrumentos (con cuchara) o causas (herido por arma blanca). Asimismo, descartamos las locuciones construidas con preposiciones livianas, como en oposición a, en relación con y las expresiones idiomáticas, tales como estar en capilla.

Analizaremos el comportamiento de estos locativos escuetos a partir de una clasificación que los agrupa en tres clases semánticas. Intentaremos demostrar que los nombres escuetos de la primera clase denotan un tipo de entidad y adquieren, por tanto, una interpretación genérica (cf. 1a). La segunda clase está integrada por escuetos de referencia individual que designan una entidad particular (cf. 1b). Por su parte, los de la tercera clase denotan una actividad prototípica que se lleva a cabo en la localización designada por el nombre escueto (cf. 1c).

Este artículo se organiza de la siguiente manera. En primer lugar, proponemos la clasificación según los tres tipos de denotación. En segundo lugar, desplegamos una serie de pruebas que nos permiten determinar la referencialidad de cada uno. En tercer lugar, analizamos las restricciones léxicas del nombre en cada grupo y el grado de productividad de cada uno de ellos. El trabajo se cierra con algunas reflexiones respecto de la tendencia del español rioplatense coloquial actual a permitir escuetos con más valores y mayor distribución de lo señalado hasta el momento. 


\section{EsCUETOS GENÉRICOS}

El primer grupo de locativos escuetos, si bien ha sido estudiado por Stvan (2009) en inglés, no cuenta con antecedentes en español. En ellos, la ausencia del determinante provoca una interpretación de clase; es decir, el nombre no denota un lugar sino un tipo de locación. Así, en (1a), no se hace referencia a una biblioteca particular sino a la clase de establecimientos designados por este nombre.

La primera prueba que nos permite dar cuenta de esta interpretación es la afinidad que muestran estos escuetos con contextos genéricos, en los que se expresan hábitos, aptitudes o tendencias y no eventos particulares (Laca 1999). De hecho, estas construcciones resultan mal formadas en contextos episódicos, como se puede observar en los contrastes de $(2 a)$ y (2b).

(2) a. El antioxidante se vende en ferretería. vs. \# El antioxidante se vendió en ferretería.

b. La ropa delicada se limpia mejor en tintorería. vs. \# La ropa delicada se limpió bien en tintorería.

La referencia a clases de estos escuetos se pone de manifiesto, también, al analizar lo que ocurre con la identidad del referente en casos de elipsis verbal y con sujetos plurales (Laca 1999; Carlson \& Sussman 2005). Ante la elipsis verbal, no se preserva la identidad de la denotación, es decir, no hay identidad estricta. En consecuencia, del ejemplo (3a) no se infiere necesariamente que el querosén y el antioxidante se venden en la misma 
ferretería. Esta falta de identidad estricta se advierte también con sujetos plurales, que permiten tanto una lectura distributiva como una no distributiva, tal como se muestra en las dos posibles continuaciones de (3b).

(3) a. El querosén se vende en ferretería y el antioxidante también.

b. Raúl y Sara estudian en biblioteca. Raúl estudia en la Biblioteca Nacional y Sara en la del Maestro. / Raúl y Sara estudian en la biblioteca del Maestro.

El tipo de modificación que pueden recibir estos escuetos es otra prueba de su interpretación genérica. Rechazan tanto modificadores como afijos que denoten propiedades atribuibles a objetos individuales, como el tamaño o la forma. Por ello, los ejemplos de (4a) con el adjetivo calificativo grande y el sufijo aumentativo -ote no son posibles (cf. Aguilar-Guevara \& Schulpen 2014: 242). En cambio, se combinan perfectamente con adjetivos relacionales y sintagmas preposicionales, que le atribuyen al nombre al que modifican una relación con una clase y no una propiedad unitaria vinculada con dimensiones físicas ( $4 \mathrm{~b})$ :

(4) a. *Este jabón de tocador se compra en supermercado grande/ supermercadote.

b. Este jabón de tocador se compra en supermercado barrial/ de barrio.

En cuanto a su comportamiento discursivo, estos escuetos no son fácilmente retomados como antecedentes. En efecto, su pronominalización en singular como recurso anafórico (5a) fuerza la lectura definida 
del antecedente en la que se retoma la referencia a una biblioteca conocida. Por el mismo motivo resulta dudoso (5b), en el que el escueto se toma como antecedente de una cláusula relativa.

Sin embargo, si el escueto genérico se retoma con el clítico en plural, la oración se torna más aceptable (5c). Pensamos que esta posibilidad de retomar el escueto con un pronombre plural podría constituir una evidencia más de su valor genérico, porque el hablante estaría reponiendo un plural (las bibliotecas), que es el modo en que se expresa la referencia a clase o tipo en español. De esta forma, en (5c) las retoma el contenido descriptivo del nombre, que no hace referencia a un individuo particular sino a un tipo de individuo. ${ }^{2}$

(5) a. ??Los alumnos estudian en biblioteca, pero Victoria la encuentra aburrida.

b. ??Los alumnos estudian en biblioteca, que siempre cuenta con bibliografía especializada.

c. Los alumnos estudian en biblioteca, pero Victoria las encuentra aburridas.

Si nos centramos en la preposición que da lugar a esta lectura de clase, observamos que se restringe a cierto tipo de preposiciones, especialmente

2 Laca (1999) afirma que las anáforas no solo son capaces de retomar referentes previamente presentados en el discurso, sino también el contenido descriptivo del nombre: "Cuando un sintagma nominal sin artículo funciona en el discurso como antecedente de un elemento anafórico, por ejemplo de un clítico o un sujeto implícito (...), estos elementos anafóricos tienen muy a menudo una referencia genérica" (Laca 1999: 896). 
a las livianas. ${ }^{3}$ De este modo, si sustituimos la preposición en de (6a-b) por su un sinónimo con claro contenido locativo como dentro de, la oración se torna agramatical, puesto que las preposiciones plenas solo son compatibles con nombres que denotan individuos y, en este caso, tintorería no refiere a un espacio particular sino a un tipo de establecimiento.

(6) a. La ropa delicada se limpia mejor en tintorería.

b. *La ropa delicada se limpia mejor dentro de tintorería, no afuera.

Lo mismo ocurre en el contraste entre (7a) y (7b): con verbos de movimiento, el valor genérico de tintorería se conserva con las preposiciones livianas a y de mientras que rechaza las versiones plenas hacia y desde.

(7) a. Siempre lleva/trae la ropa fina a/de tintorería.

b. *Siempre lleva/trae la ropa fina hacia/desde tintorería.

En lo que concierne al grado de productividad de estas construcciones con valor genérico en español, hemos observado que existen restricciones léxicas, esto es, los nombres que las conforman pertenecen a una clase acotada. Solo son posibles con nombres locativos que denotan establecimientos, generalmente comerciales (8a), y no con otro tipo de lugares,

3 Adoptamos la distinción entre preposiciones plenas, que son las que tienen contenido semántico (como desde y contra), y las preposiciones livianas, que funcionan como palabras gramaticales, en tanto no aportan significado conceptual alguno (cf. Masullo 1996). Ašic \& Corblin (2012) se refieren a estas últimas como "preposiciones locativas menos especificadas" (less specified locative prepositions). 
como pueden ser las partes de esos establecimientos o de una vivienda (8b). Tampoco admiten nombres que denotan espacios naturales (8c).

(8) a. Esto se vende en librería/ panadería/ frutería/ zapatería/ supermercado/ carnicería.

b. *Este mueble se pone en pasillo/ cocina/ en dormitorio/ en baño.

c. *Este animal vive en llanura/ selva/ estepa/montaña.

Así pues, este primer grupo de escuetos se caracterizan por referir a tipos de entidades. Hemos visto cómo esta interpretación genérica se pone en evidencia por su afinidad con contextos genéricos, por el hecho de que tanto en casos de elipsis verbal como con sujetos plurales es posible una lectura en que no hay identidad estricta y por el tipo de modificación que admiten. En cuanto a la posibilidad de anáfora, hemos mostrado que no son buenos candidatos para introducir referentes en el discurso y que los nombres que pueden intervenir en estas construcciones pertenecen a una clase de locativos que denotan un tipo específico de establecimiento.

\section{ESCUETOS DE REFERENCIA INDIVIDUAL}

El segundo grupo de locativos estudiados se diferencia del primero en que en estos casos los singulares escuetos denotan entidades individuales. Así, en el ejemplo (1b) rectorado denota un lugar concreto. Veremos que estos escuetos también se construyen a partir de una clase léxica restringida y que presentan dos implicaturas: una de familiaridad -similar a la trabajada por Stvan (2009) en inglés- y una de unicidad. 
En primer término, en contraste con lo visto en los genéricos, estos escuetos pueden aparecer tanto en contextos genéricos como en episódicos, si bien su valor de individuo se pone de manifiesto sobre todo en contextos que expresan eventos particulares y puntuales, como se puede observar en los ejemplos de (9):

(9) a. La auditora siempre atiende en tesorería/ Hoy la auditora nos atendió en tesorería.

b. Mi hermana trabaja en secretaría/ Estuve en secretaría toda la tarde.

En segundo término, como es de esperar, estos escuetos preservan la identidad de su denotación. Por lo tanto, mantienen la identidad estricta con su antecedente ante casos de elipsis verbal. De este modo, la única interpretación posible de (10a) es que Sara y Facundo están en el mismo lugar. Por el mismo motivo, con sujetos plurales no admiten una lectura distributiva, es decir, en (10b) la única interpretación posible es que tanto Sara como Facundo se hallan en el mismo lugar (Sara y Facundo están en la bedelía de su universidad). No es posible inferir que Sara se encuentra en una bedelía y Facundo en otra (\#Sara está en la bedelía de la Universidad de la República y Facundo en la de la Universidad de Buenos Aires).

(10) a. Sara está en bedelía y Facundo también. ${ }^{4}$

b. Sara y Facundo están en bedelía.

4 Bedelía $f$ En los centros de estudios, oficina para la atención y trámites de los estudiantes (Kühl de Mones 1993). 
Puesto que denotan entidades individuales, este tipo de escuetos no admite la pluralización. Como se ve en (11a), al pluralizar el nombre, la oración resulta anómala. La única manera de rescatarla sería mediante un contexto que hiciera posible su interpretación como un plural escueto con valor de tipo de entidad (Laca 1999), como se muestra en (11b).

(11) a. \#La auditora nos atendió en tesorerías.

b. La auditora suele hacer inspecciones en tesorerías.

Su comportamiento discursivo constituye otra prueba de la capacidad de estos escuetos de referir a un lugar individual existente. En efecto, sirven para introducir referentes en el discurso y por lo tanto pueden ser perfectamente retomados mediante un pronombre, como se muestra en (12), en donde el clítico la remite a la locación en la que se encuentra el sujeto.

(12) Ya estoy en bedelía; la encontré desierta.

Por otra parte, y a pesar de tener carácter referencial, estos escuetos no suelen aparecer modificados, ni por adjetivos calificativos o relacionales ni por sintagmas preposicionales (13).

(13) *El trámite lo hice en bedelía nueva/ universitaria/ de la escuela.

La explicación de la incompatibilidad con modificadores la encontramos en que en estos casos hay una implicatura de unicidad, es decir, que el nom- 
bre escueto denota un lugar individual que es único en el ámbito en el que se encuentra el hablante. De hecho, de reponer un determinante sin alterar el valor semántico, esta construcción solo admitiría un determinante definido fuerte, que habilita la lectura de unicidad; nunca un indefinido (14).

(14) En este momento, el decano está en rectorado/ en el rectorado/ *en un rectorado.

La implicatura de unicidad está asociada a su vez a la implicatura de familiaridad que tienen estos escuetos, ya que denotan siempre un lugar identificable por hablante y oyente. Este valor, que Stvan (2009) denomina "deíctico", es lo que explica que estos locativos se interpreten tomando como punto de anclaje el lugar en el que está la persona denotada por el sujeto de la oración. Así, de (15a) se infiere que Agustín trabaja en el rectorado de su universidad y de (15b), que los subgerentes estuvieron en la secretaría de su lugar de trabajo.

(15) a. Agustín trabaja en rectorado.

b. Los subgerentes se reunieron en secretaría durante toda la tarde.

El carácter de "único", que adquiere el nombre escueto y que permite asimilarlo a un nombre propio, fue estudiado en las gramáticas como un rasgo dialectal. De hecho, ya en la Sintaxis Hispanoamericana (Kany 1970 [1945]) se alude a casos similares. ${ }^{5}$ Este fenómeno, que Kany des-

5 "Con unos pocos nombres, como casa, palacio, etc., el español corriente omitió el artícu- 
cribe como ya en desuso, es muy frecuente en las variedades rioplatenses habladas tanto en Argentina como en Uruguay y permite comprender el comportamiento de estos escuetos en combinación con cláusulas relativas. En efecto, como se observa en los ejemplos de (16), solo admiten ser modificados por oraciones subordinadas relativas no restrictivas (16a), porque estas agregan información acerca de la entidad denotada por el nombre que funciona como antecedente, y rechazan las que tienen valor especificativo (16b), dada la imposibilidad de especificar una entidad única. El mismo comportamiento se observa con los nombres propios (17).

(16) a. El trámite lo hice en bedelía, que está en primer piso.

b. *El trámite lo hice en bedelía que está en primer piso.

(17) a. Se mudó a Rosario, que está en la Provincia de Santa Fe.

b. *Se mudó a Rosario que está en la Provincia de Santa Fe.

Este comportamiento, que los asemeja a los designadores rígidos, se advierte además en el hecho de que pueden ocupar posición de sujeto y de objeto sin determinante, manteniendo su valor locativo (18), lo cual en español solo es posible con nombres propios de lugar (19).

lo definido desde antiguo, posiblemente porque "el nombre fue considerado originalmente como único y de hecho como nombre propio” (...). Dicho uso se extendió en algunos casos al espańol americano, pero generalmente ha caído en desuso (Kany 1970 [1945]: 39). 
(18) a. Rectorado queda en planta baja.

b. ¿Ubicás rectorado?

(19) a. Rosario queda cerca.

b. ¿Ubicás Rosario?

De modo análogo a lo observado en los nombres escuetos genéricos, los locativos que participan de estas construcciones presentan restricciones léxico-semánticas, en la medida en que denotan locaciones únicas que se reconocen como parte de una institución. Por este motivo, son posibles los ejemplos de (20a) pero no los de (20b). En (20a), tesorería hace referencia a un espacio que forma parte de una dependencia pública o privada. En (20b), dormitorio, si bien denota una parte de una vivien$\mathrm{da}$, no admite esta lectura porque no integra un establecimiento organizado en partes institucionalizadas. En el caso de oficina, tampoco se cumple la implicatura de unicidad, ya que usualmente las instituciones cuentan con más de una oficina.

(20) a. El contador se encuentra en tesorería.

b. *El contador se encuentra en dormitorio/ en oficina.

Así pues, estas construcciones -bastante productivas en el español rioplatense- se pueden aplicar a cualquier nombre locativo, siempre y cuando este denote una dependencia institucional. En (21a) dirección denota el lugar destinado al director de la institución en la que se encuentra el hablante y en (21b) y (21c) sala de profesores y secretaría académica se 
interpretan como partes de instituciones educativas. Adviértase que en ambos casos el nombre locativo está modificado, ya sea por un sintagma preposicional (21b) o por un adjetivo relacional (21c). Esto, que en principio parece contradecir la implicatura de unicidad planteada para estos locativos, en realidad no lo hace, puesto que en estos casos las partes de la institución referidas llevan los nombres de sala de profesores y secretaría académica respectivamente.

(21) a. Me citaron en dirección.

b. El listado de asistencia está en sala de profesores.

c. El formulario se entrega en secretaría académica.

El tipo de referencialidad que conllevan estos escuetos explica también su comportamiento en relación con la posibilidad de sustitución por otras preposiciones locativas. Al mantener su denotación locativa individual permiten la permutación con cualquiera de ellas, como se ilustra en (22), sin importar que se trate de preposiciones plenas (22a) o livianas (22b).

(22) a. Estoy dentro de rectorado./ Voy hacia rectorado./ Vengo desde rectorado.

b. Estoy en rectorado./ Voy a rectorado./ Vengo de rectorado.

En suma, este segundo grupo, el de los escuetos de referencia individual, denota entidades particulares, como se puede advertir por su posibilidad de aparición en contextos episódicos, por el hecho de que 
mantienen identidad estricta en casos de elipsis verbal, por el valor semántico que adquieren con la pluralización y por su comportamiento anafórico. Además de este valor referencial, poco usual para los escuetos contables singulares en español, encontramos una serie de implicaturas asociadas. Por un lado, conllevan una implicatura de unicidad que se manifiesta en el tipo de modificación y de cláusulas relativas que admiten, similar a la de los nombres propios. Por otro lado, la locación debe ser familiar para hablante y oyente. Finalmente, relevamos las restricciones léxicas vinculadas con esta lectura, que surgen de que estos escuetos denotan locaciones únicas e identificables que forman parte de una institución.

\section{EsCuetos DE ACTIVIDAD}

El tercer grupo está conformado por secuencias en las que el nombre escueto denota una actividad prototípica que se lleva a cabo en la locación denotada por el nombre. Así, en el ejemplo (1c), estar en quirófano se interpreta como 'estar realizando una intervención quirúrgica en un quirófano'.

Estos escuetos, que existen tanto en italiano (a scuola) como en inglés (at school), han sido más estudiados en las lenguas germánicas (Stvan 2009; Le Bruyn et al 2011). En español se suelen mencionar la forma lexicalizada estar en cama, que tiene la lectura de 'estar en la cama padeciendo una enfermedad' (23a) y la construcción estar en prisión, que se entiende como 'estar en una prisión cumpliendo una condena'(23b) (RAE/ASALE 2009; Laca 1999). 
(23) a. Juan no puede salir porque está en cama.

b. Estudió abogacía en prisión.

En tanto forma lexicalizada, estar en cama ha perdido transparencia semántica; de hecho, la versión con determinante (estar en una cama) no se interpreta necesariamente como 'estar enfermo'. Por el contrario, la lectura de actividad asociada a estar en prisión se puede inferir del significado de prisión. Asimismo, hemos visto que esta lectura de actividad no solo se aplica a más casos de los tradicionalmente estudiados sino que, a diferencia de las otras dos construcciones con escuetos, esta presenta una productividad diferente en ambas orillas del Río de la Plata, como se verá más abajo.

Consideramos que el enriquecimiento semántico que ocurre en estos casos se debe a que la ausencia del determinante produce un cambio del dominio conceptual del nombre, que pasa de denotar una entidad concreta a denotar un proceso. La posibilidad de obtener esta interpretación está estrechamente vinculada con el carácter funcional de los nombres involucrados, es decir, con el hecho de que prisión, ruta, consultorio no denotan clases naturales sino que son artefactos. Según Pustejovsky (1995), los nombres funcionales incorporan aspectos semánticos que los vinculan con eventos típicos asociados a su función, que forman parte de lo que este autor llama el quale télico. ${ }^{6}$

6 Ašic \& Corblin (2012) analizan el carácter funcional de los definidos débiles (telic definites) en construcciones locativas del francés y del serbio. Zwarts (2014), por su parte, incorpora el concepto de rol télico de Pustejovsky (1995) y postula una operación de framing a partir de la cual la información funcional se recupera cuando se le asigna a un nombre un rol en un marco (frame). 
De este modo, en (24) se activan sentidos introducidos por este quale, que forman parte de la configuración semántica de los nombres involucrados. En el primer ejemplo se recupera el significado de la actividad típica de atender pacientes, normalmente llevada a cabo en un consultorio, de modo que (24a) se lee como 'Te llamo más tarde, ahora estoy atendiendo pacientes en un consultorio'. En el segundo ejemplo se remite a la actividad de andar o manejar en una ruta, con lo cual (24b) se interpreta como 'Pedro no habla por celular cuando está manejando en una ruta'.

(24) a. Te llamo más tarde, ahora estoy en consultorio.

b. Pedro no habla por celular cuando está en ruta.

Al formar parte de la estructura semántica de los nombres funcionales, estos sentidos también están presentes en otros contextos sintácticos, como se muestra en (25). Por ello, en (25a), la ruta se interpreta como 'manejar o andar en una ruta' y en (25b), el quirófano y el consultorio reciben las lecturas de 'operar a alguien en un quirófano' y de 'atender pacientes en un consultorio'.

(25) a. La ruta lo cansó mucho.

b. El Dr. Huertas prefiere el quirófano al consultorio.

Proponemos una serie de pruebas para dar cuenta de la lectura de actividad. La primera consiste en mostrar que estas construcciones solo aceptan sujetos animados, ya que las actividades implicadas suponen un 
participante humano. ${ }^{7}$ Como se ve en los ejemplos, (26a) y (26b) admiten lecturas de actividad: 'La Dra. Bruno está atendiendo pacientes'; 'Sus hijos están asistiendo a clases en un jardín de infantes'. En (26c) y (26d), en cambio, los sujetos no animados no admiten construcciones con locativos escuetos.

(26) a. La Dra. Bruno está en consultorio.

b. Sus hijos están en jardín de infantes.

c. *La camilla quedó en consultorio.

d. *Los crayones están en jardín de infantes.

Por otro lado, si sometemos estos escuetos a la prueba de elipsis verbal, observamos que el nombre locativo no es referencial. En contraste con lo que ocurre con los escuetos de referencia individual, en estos casos no es posible establecer una identidad estricta. En (27a) las únicas interpretaciones posibles son que Juan y Mario están manejando en una ruta, y en (27b), que tanto la Dra. Bruno como el Dr. Alonso están atendiendo pacientes en un consultorio. Es decir que, como es de esperar, lo que se retoma al restituir la elipsis son las actividades asociadas a los locativos ruta y consultorio y no los espacios designados por estos nombres.

(27) a. Juan está en ruta y Mario también.

b. La Dra. Bruno está en consultorio y el Dr. Alonso también.

7 Nótese como excepción el caso de en taller, citado por Laca (1999: 923), en que el sujeto es el objeto afectado por la actividad; el auto está en taller se entiende como 'el auto está en reparación'. 
El hecho de que estos escuetos no designan espacios particulares, sino las actividades asociadas a ellos, permite también explicar la anomalía en (28). La marca morfológica plural de estos nombres no tiene como correlato la designación de un conjunto de entidades, así como tampoco designa una única entidad la marca singular. Esto es así porque resulta irrelevante determinar cuántas rutas está recorriendo Juan o en cuáles quirófanos operó la Dra. Bruno, pues lo que se pone de relevancia son las actividades desempeñadas por ambos participantes.

(28) a. \# Juan está en rutas.

b. \# La Dra. Bruno estuvo en quirófanos.

La tercera prueba se relaciona con la modificación. En las construcciones que se ilustran en (29a) y (29b) resulta agramatical combinar estos escuetos con algún tipo de modificador, ya sea de referencia individual o de clase. Esto se debe a que estos nombres no denotan entidades concretas sino actividades y, por lo tanto, no se les puede atribuir propiedades. Sin embargo, admiten modificadores atélicos tales como sintagmas preposicionales encabezados por durante (29c), en los que se refuerza la naturaleza no delimitada de las actividades designadas por los nombres.

(29) a. *Juan está en cama grande/ ortopédica.

b. *No te puedo hablar porque estoy en ruta peligrosa/ nacional.

c. Juan estuvo en cama durante un mes.

La cuarta evidencia que señala su carácter de actividad, y por tanto no referencial, está vinculada con las oraciones relativas. Como se ilus- 
tra en (30), estos escuetos rechazan las cláusulas relativas, tanto restrictivas como especificativas. En (30a) el pronombre relativo es incapaz de seleccionar el escueto como antecedente, puesto que las oraciones subordinadas solo modifican expresiones que denotan una entidad (Espinal \& McNally 2011). Asimismo, la agramaticalidad de (30b) está dada porque el participio modifica a un nombre que no constituye una expresión referencial, sino que refiere a una actividad.

(30) a. *Estamos en ruta que queda al sur de la provincia.

b. *Estudió abogacía en prisión, ubicada en las afueras de la capital.

Estos nombres escuetos tampoco tienen valor presentativo, esto es, no pueden ser retomados en el discurso. En consecuencia, el problema de (31a) es que el pronombre ella es incapaz de retomar el valor de actividad de cama. Análogamente, la continuación de (31b) resulta inadecuada, porque el adverbio ahi exige un antecedente con significado locativo y en este caso quirófano no denota una locación sino una actividad.

(31) a. \# Juan está en cama y se va a quedar en ella hasta que se cure.

b. La Dra. Bruno está en quirófano. \# La Dra. Bruno está ahí.

Asimismo, la interpretación de actividad de estas construcciones explica su comportamiento con respecto a la imposibilidad de sustituir la preposición en por otras preposiciones, ya sea plenas o livianas. Como en estos casos los nombres no refieren a una entidad particular, no aceptan ser combinados con otras preposiciones, como muestra la agramaticalidad de las oraciones en (32). 
(32) a. *María viene de consultorio.

b. *Juan está dentro de prisión por fraude.

Como adelantáramos previamente, un fenómeno interesante de resaltar es que este tipo de escuetos muestra una productividad mayor en el español uruguayo que en el argentino. Así, los ejemplos de (33), que son rechazados por hablantes de la Argentina, resultan normales en Uruguay:

(33) a. Es más emocionante ver los partidos cuando son en estadio.

b. Los niños están en piscina lunes y miércoles de 5 a 7 .

c. A partir de las 5 no me llames porque estoy en gimnasio.

d. Pedro está en laboratorio todo el día.

e. Llego a la reunión porque a esa hora ya no estoy en facultad.

En todos estos casos, los sintagmas preposicionales escuetos son interpretados como una actividad prototípica que se lleva a cabo en la locación que el nombre en cuestión designa. De esta manera, en (33a) se entiende que los jugadores están en el estadio jugando al fútbol; en (33b), que los niños van a clase de natación lunes y miércoles de 5 a 7 ; en (33c), que a partir de las 5 de la tarde hago gimnasia en el gimnasio; en (33d), que Pedro trabaja en el laboratorio todo el día y en (33e), que llego a la reunión porque a esa hora ya no estoy estudiando en la facultad.

Este fenómeno de microvariación parece hacer sistema con los escuetos singulares contables que ocurren en posición de objeto directo estudiados en Oggiani (2013) en el español del Uruguay. En los ejemplos de 
(34), que tampoco son posibles en el español de de Argentina, los nombres refieren a entidades individuales.

(34) a. Papá está haciendo parrillero.

b. Cecilia termina monografía y se recibe.

A modo de resumen, este tipo de construcciones con contables escuetos singulares se interpretan como una actividad prototípica que ocurre en el lugar que el nombre denota. En primer lugar, hemos planteado que solo aceptan sujetos animados con el rasgo [+humano]. Luego, verificamos su falta de referencialidad mediante una serie de pruebas: su comportamiento ante la elipsis verbal y la pluralización, su incompatibilidad con modificadores adjetivales, el rechazo de subordinadas relativas, la imposibilidad de retomarlos anafóricamente mediante pronombres o de reemplazar en por otras preposiciones. Asimismo, observamos las restricciones léxicas de estos nombres y el fenómeno de microvariación que presentan.

\section{CASOS DE AMbigüEDAD}

Es posible advertir casos ambiguos en los distintos tipos de escuetos analizados. Por un lado, existen casos en los que es posible tanto una lectura genérica como una de referencia individual (35).

(35) a. Este antigripal se consigue en farmacia. Lo podés comprar tanto en la farmacia del shopping como en la de la esquina.

b. Este antigripal se consigue en farmacia, que abre a las $9 \mathrm{hs.}$ 
En (35a), farmacia se puede interpretar como un genérico: 'Este antigripal se consigue en cualquier farmacia'. Esta lectura está habilitada por el contexto genérico afín, aportado por el tiempo presente del verbo y porque al referir a un tipo de establecimiento comercial, el nombre se puede sustituir por un plural escueto con el mismo valor (Este antigripal se consigue en farmacias). Pero también admite una segunda interpretación, según la cual el antigripal se consigue en la sección destinada a la farmacia de un establecimiento en el que se encuentra el hablante. A diferencia de la lectura anterior, aquí la referencia individual de farmacia admite la modificación de una cláusula relativa no restrictiva (35b).

El segundo caso de ambigüedad se da entre la lectura de referencia individual y la lectura de actividad (36). Así, en (36a) podemos interpretar que la doctora se encuentra en el quirófano de la clínica en la que trabaja o que está realizando una intervención quirúrgica. De la primera interpretación surgen las implicaturas de familiaridad y unicidad, mediante las cuales hablante y oyente comparten el conocimiento de que hay un solo quirófano en el lugar en donde se encuentran. En la primera lectura, el carácter referencial del nombre hace posible su pronominalización (36b), que es imposible en la interpretación de actividad, en la que la anáfora lo no puede retomar el valor de actividad de quirófano.

(36) a. La doctora está en quirófano.

b. La doctora está en quirófano, lo encontrás en el quinto piso.

No es de extrañar que no se encuentren casos de ambigüedad entre una lectura genérica y una de actividad. Como hemos visto, los nombres 
escuetos que pueden producir una lectura genérica denotan una clase acotada de locativos, la de los establecimientos institucionales o comerciales, y los nombres analizados que admiten la interpretación de actividad pertenecen a esta clase. Naturalmente, los casos ambiguos responden al hecho de que los locativos involucrados pertenecen a las clases léxicas propias de cada grupo. Así, la ambigüedad entre la interpretación genérica y la de referencia individual es posible con farmacia, porque este nombre puede denotar tanto un tipo de establecimiento como un lugar específico que forma parte de una institución. De manera similar, quirófano admite una lectura de referencia individual además de la de actividad prototípica que se asocia al nombre en virtud de su denotación. Este comportamiento ambiguo no es aplicable a nombres locativos como ruta y cama, que solo se interpretan como actividades, ya que es imposible tomarlos como locaciones únicas que forman parte de una institución. Queda claro, entonces, que la misma naturaleza léxica de estos nombres escuetos determinará los casos en los que pueden recibir más de una interpretación.

\section{Conclusiones}

En este trabajo hemos analizado una serie de construcciones locativas del español, introducidas por la preposición en. El estudio realizado nos permitió reconocer tres grupos diferentes, que se distinguen por el valor semántico que adquiere el nombre locativo en el español rioplatense. Una de las principales diferencias entre ellos radica en el tipo de referencialidad. En el primer grupo, el de los escuetos genéricos, los nombres 
escuetos denotan tipos de locación; en el segundo, los escuetos refieren a locaciones individuales. En el tercer grupo, en cambio, los nombres involucrados carecen de carácter referencial.

Los escuetos locativos con interpretación genérica no han sido estudiados en el español. Como hemos visto, la lectura de clase en construcciones solo es posible con un conjunto restringido de nombres: aquellos que denotan tipos de establecimiento (en biblioteca, en zapatería, en ferretería).

Los escuetos de referencia individual, por su parte, son particularmente productivos en el español rioplatense. Es interesante advertir que, además de tener la capacidad de denotar locaciones individuales, estos escuetos involucran dos implicaturas: denotan un lugar único (implicatura de unicidad) y deben remitir a un lugar conocido por hablante y oyente (implicatura de familiaridad). En cuanto a las restricciones léxico-semánticas, siempre refieren a partes reconocibles de un establecimiento público o privado (en rectorado, en secretaría, en sala de profesores).

Finalmente, los escuetos de actividad se distinguen de los otros dos grupos en que no refieren ni a tipos de locaciones ni a lugares individuales. Como hemos visto, en estos casos la ausencia de determinante genera un enriquecimiento semántico por el cual estas construcciones se interpretan como actividades prototípicas llevadas a cabo en el lugar denotado por el nombre (en ruta, en quirófano). En estos casos también encontramos restricciones léxico-semánticas, ya que solo se producen con nombres locativos que se pueden asociar a actividades prototípicas, es decir, a nombres que tienen asociado un evento en su quale télico. Lo más interesante de estos escuetos es que presentan un mayor grado de productividad en Uruguay que en Argentina. 
Las restricciones léxico-semánticas observadas en los tres grupos explican que los casos de ambigüedad se den solo entre las lecturas genérica e individual (en farmacia) o entre la de referencia individual y la de actividad (en quirófano).

Por otro lado, cabe preguntarse por qué un hablante decide omitir el determinante en cada una de estas construcciones cuando tiene otras opciones: el plural escueto con valor genérico (Estudia en bibliotecal bibliotecas); el determinante definido para el referencial (Está en bedelíal en la bedelía) y el verbo en lugar de los escuetos de actividad (No atiende el teléfono cuando está en consultorio/ está atendiendo pacientes). En cuanto al genérico, si bien no podemos responderlo a cabalidad, advertimos, a partir de los datos analizados que su uso podría estar vinculado con cuestiones de índole discursiva, en la medida en que resultan malos candidatos para introducir referentes a nivel discursivo. En los de referencia individual, en cambio, además de la preferencia dialectal que reflejan, tanto la restricción léxica como las implicaturas permiten establecer condiciones claras de diferenciación con sus variantes con determinante. Por fin, la interpretación de actividad que surge por la ausencia de determinante se aplica especialmente a nombres que denotan locaciones factibles de asociarse a una actividad prototípica. En ellos es particularmente interesante la microvariación observada entre las dos variedades rioplatenses.

Los valores semánticos estudiados nos permiten concluir que estos casos, lejos de constituir excepciones, parecieran representar una tendencia en el español rioplatense coloquial a permitir nombres escuetos con una gama más amplia de denotaciones y una mayor distribución de lo que sería esperable. En efecto, resulta evidente que la ausencia de 
determinante en esta variedad dialectal cubre más usos que los consignados en la literatura clásica. En este sentido, la productividad de los escuetos locativos que hemos analizado, se suma a la posibilidad que tienen de ocupar posiciones argumentales de objeto, como en escribir tesis o terminar monografía, estudiados en Oggiani (2013) y a los que aparecen en construcciones como pegar novia o pintar beso, estudiadas por Kornfeld \& Kuguel (2013).

\section{REFERENCIAS}

Aguilar-Guevara, Ana \& Schulpen, Maartje. 2014. Modified weak definites. En Aguilar-Guevara, Ana \& Le Bruyn, Bert \& Zwarts, Joost (eds.), Weak referentiality, 237-264. Ámsterdam: John Benjamins. Ašic, Tijana \& Corblin, Francis. 2012. Telic definites and their preposition(s): French vs. Serbian. (Ponencia presentada en el congreso 'Languages with and without articles', París, marzo de 2012.)

Baldwin, Timothy \& Beavers, John \& Van Der Beek, Leonoor \& Bond, Francis \& Flickinger, Dan \& Sag, Ivan A. 2006. In search of a systematic treatment of determinerless Pps. En Saint-Dizier, Patrick (ed.), Syntax and semantics of prepositions, 163-179. Dordrecht: Springer. Bosque, Ignacio (ed.). 1996. El sustantivo sin determinación. La ausencia de determinante en la lengua española. Madrid: Visor.

Carlson, Greg \& Sussman, Rachel. 2005. Seemingly indefinite definites. En Kepsar, Stephan \& Reis, Marga (eds.), Linguistic evidence: Empirical, theoretical, and computational perspectives, 71-86. Berlín: Mouton de Gruyter. 
Carlson, Greg \& Sussman, Rachel \& Klein, Natalie \& Tanenhaus, Michael. 2006. Weak definite NPs. En Davis, Christopher \& Deal, Amy Rose \& Zabbal, Yourl (eds.), Proceedings of the thirty-sixth annual meeting of the North East Linguistics Society, 179-196. Amherst: GLSA Publications.

Corblin, Francis. 2011. Des définis para-intensionnels: être à l'hôpital, aller à l'école. Langue française 171. 55-77.

Espinal, María Teresa. 2010. Bare nominals in Catalan and Spanish: Their structure and meaning. Lingua 120. 984-1009.

Espinal, María Teresa \& McNally, Louise. 2011. Bare nominals and incorporating verbs in Spanish and Catalan. Journal of Linguistics 47. 87-128.

Kany, Charles. 1970 [1945]. Sintaxis hispanoamericana. Madrid: Gredos. Kühl de Mones, Úrsula. 1993. Nuevo diccionario de uruguayismos. En Haensch, Günther \& Werner, Reinhold (dirs.), Nuevo diccionario de americanismos, t. III. Santa Fe de Bogotá: Instituto Caro y Cuervo. Kornfeld, Laura \& Kuguel, Inés. 2013. Pegar laburo y pintar bardo: procesos de gramaticalización y lexicalización en el ámbito verbal. En Kornfeld, Laura \& Kuguel, Inés (eds.), El español rioplatense desde una perspectiva generativa, 95-112. Mendoza: Facultad de Filosofía y Letras / Universidad Nacional de Cuyo / Sociedad Argentina de Lingüística. Laca, Brenda. 1999. Presencia y ausencia de determinante. En Bosque, Ignacio \& Demonte, Violeta (eds.), Gramática descriptiva de la lengua española, vol. 1, 891-921. Madrid: Espasa Calpe.

Le Bruyn, Bert \& De Swart, Henriette \& Zwarts, Joost. 2011. Mass-count distinctions in bare PPs. Utrecht: Utrecht University. (manuscrito.) 
http://www.let.uu.nl/users/Joost.Zwarts/personal/Masscount.pdf. (Consultado el 24/05/2016.)

Masullo, Pascual. 1996. Los sintagmas nominales sin determinante: una propuesta incorporacionista. En Bosque, Ignacio (ed.), El sustantivo sin determinación. La ausencia de determinante en la lengua española, 169-200. Madrid: Visor.

Oggiani, Carolina. 2013. Una propuesta de self paced-reading para analizar los nombres escuetos contables en posición de objeto. (Ponencia presentada en VI Encuentro de Gramática Generativa, Universidad Nacional del Comahue, Argentina, 8-10 de agosto de 2013.)

Pustejovsky, James. 1995. The generative lexicon. Cambridge: MIT Press. Real Academia Española \& Asociación de Academias de la Lengua Española. 2009. Nueva gramática de la lengua española. Madrid: Espasa. Stvan, Laurel S. 2009. Semantic incorporation as an account for some bare singular count noun uses. Lingua 119(2). 314-333.

Zwarts, Joost. 2014. Functional frames in the interpretation of weak nominals. En Aguilar-Guevara, Ana \& Le Bruyn, Bert \& Zwarts, Joost (eds.), Weak referentiality, 265-286. Ámsterdam: John Benjamins.

Fecha de recepción de manuscrito original: 22 de diciembre de 2015 Fecha de recepción de manuscrito revisado: 12 de mayo de 2016 Fecha de aceptación: 12 de mayo de 2016 